\title{
The efficacy of treatment options for patients with gastric cancer and peritoneal metastasis
}

\author{
Beate Rau $^{1} \cdot$ Andreas Brandl $^{1}(0)$ Peter Thuss-Patience ${ }^{2} \cdot$ Fabian Bergner $^{1} \cdot$ Wieland Raue $^{3} \cdot$ Alexander Arnold $^{4}$. \\ David Horst ${ }^{4}$. Johann Pratschke ${ }^{1}$. Matthias Biebl ${ }^{1}$
}

Received: 6 October 2018 / Accepted: 29 April 2019 / Published online: 7 May 2019

(c) The International Gastric Cancer Association and The Japanese Gastric Cancer Association 2019

\begin{abstract}
Background Patients with peritoneal metastases of gastric cancer have a poor prognosis and median survival of 7 months. This study compared treatment options and outcomes based on the Peritoneal Cancer Index (PCI).

Methods This retrospective analysis included patients with gastric cancer treated between August 2008 and December 2017 with synchronous peritoneal metastases only diagnosed by laparoscopy. The three treatments were as follows: (1) cytoreductive surgery (CRS) and hyperthermic intraperitoneal chemotherapy (HIPEC) in combination with pre- and postoperative systemic chemotherapy $(n=58)$, (2) laparotomy/laparoscopy without CRS, but HIPEC in combination with pre- and postoperative systemic chemotherapy $(n=11)$, and (3) systemic chemotherapy only $(n=19)$.

Results A total of 88 patients aged $54.6 \pm 10.9$ years with mean PCI of $14.3 \pm 11.3$ were included. The PCI was significantly lower in group $1(8.3 \pm 5.7)$ than in group $2(23.9 \pm 11.1, p<0.001)$ and group $3(27.3 \pm 9.3, p<0.001)$. Mean time from diagnosis to laparoscopy was $5.2 \pm 2.9$ months. The median overall survival was $9.8 \pm 0.7$ for group $1,6.3 \pm 3.0$ for group 2 and $4.9 \pm 1.9$ months for group $3(p<0.001)$. Predictors for deteriorated overall patient survival included $>4$ cycles of preoperative chemotherapy (HR 4.49, $p<0.001$ ), lymph-node metastasis (HR 3.53, $p=0.005), \mathrm{PCI} \geq 12$ (HR 2.11, $p=0.036$ ), and incompleteness of cytoreduction (HR 4.30, $p=0.001$ ) in patients treated with CRS and HIPEC.

Conclusion CRS and HIPEC showed convincing results in selected patients with $\mathrm{PCI}<12$ and complete cytoreduction. Prolonged duration ( $>4$ cycles) of preoperative intravenous chemotherapy reduced patient survival in patients suitable for CRS and HIPEC.
\end{abstract}

Keywords Peritoneal metastases · Gastric cancer · Hyperthermic intraperitoneal chemotherapy · Cytoreductive surgery · Preoperative chemotherapy

\section{Introduction}

Beate Rau and Andreas Brandl have contributed equally to this work.

Beate Rau

Beate.Rau@charite.de

1 Department of Surgery, Campus Virchow-Klinikum and Charité Campus Mitte, Charité-Universitätsmedizin Berlin, Augustenburger Platz 1, 13353 Berlin, Germany

2 Medical Clinic for Hematology, Oncology and Tumor-Immunology, Campus Virchow-Klinikum, Charité-Universitätsmedizin Berlin, Berlin, Germany

3 Department of General, Visceral and Thoracic Surgery, AKH Celle, Celle, Germany

4 Institute of Pathology, Charité-Universitätsmedizin Berlin, Berlin, Germany
Although the incidence of gastric cancer in Europe is decreasing, the mortality from it remains high. Overall, $17 \%$ of patients with gastric cancer show peritoneal metastases at the time of diagnosis [1]. In addition, the prognosis of patients with stage IV gastric cancer is poor with a median overall survival of 7 months after diagnosis and best supportive care [2].

Based on Lauren's histological classification of gastric carcinoma, many histopathologic subgroups for the development of metachronous peritoneal metastasis have been identified and used as predictive factors. One study found that of the patients with a diffuse subtype of gastric cancer, $80 \%$ had peritoneal metastases, in contrast to patients with the intestinal subtype (40\%) [3]. Further predictive factors 
such as infiltration of the serosa, lymph-node positive status, signet ring cell cancer, and undifferentiated grading have been described [4].

The treatment options for patients with peritoneal metastases of gastric cancer are limited and include palliative systemic chemotherapy and/or best supportive care according to the National Comprehensive Cancer Network $(\mathrm{NCCN})$ guidelines [5].

In recent years, multimodal treatment options including cytoreductive surgery (CRS) and hyperthermic intraperitoneal chemotherapy (HIPEC) had been developed to improve overall survival in selected patients. Several studies confirmed the efficacy of these treatments for patients with peritoneal metastases from gastric cancer [6]. The most important predictive factors are the completeness of cytoreduction and a Peritoneal Cancer Index $(\mathrm{PCI}) \leq 6[6$, 7]. The PCI was created by Jacquet and Sugarbaker, and is commonly used to quantitatively describe the size and area of peritoneal metastases from various gastrointestinal tumors or primary peritoneal surface malignancies [8] (Fig. 1a). Patients with a PCI $>6$ or PCI $>12$ do not have any survival benefit from CRS and HIPEC according to several publications $[7,9]$.

In terms of survival outcome, the best systemic chemotherapy regimen for patients with metastasized gastric cancer is a combination of 5-FU, oxaliplatin, and docetaxel, or fluoropyrimidine, cisplatin plus trastuzumab in those with HER2-receptor overexpression [10, 11]. A recent trial by Al-Batran et al. was able to prove superiority of neoadjuvant docetaxel, oxaliplatin, fluorouracil, and leucovorin (FLOT) compared to treatment with epirubicin, cisplatin, and fluorouracil or capecitabine (ECF/ECX) in patients with resectable gastric cancer [12]. There are currently no data on the duration of preoperative systemic chemotherapy for patients suitable for CRS and HIPEC. The standard intravenous treatment regimen is used by analogy to the published ACCORD and MAGIC trial with the purpose of downsizing, 3-6 cycles before re-evaluation for CRS and HIPEC $[13,14]$.

The aim of the present study was to compare several treatment options delivered at our center over a 9-year period and patient outcome after CRS and HIPEC based on the preoperative chemotherapy regimen.

\section{Materials and methods}

\section{Patient selection}

This explorative, retrospective study included consecutive patients diagnosed with synchronous peritoneal metastases of gastric cancer confirmed through a biopsy between August 2008 and December 2017. Every patient was evaluated, and therapeutic options were discussed in the weekly oncological conference for peritoneal surface malignancies of the multidisciplinary team (MDT) (surgical oncologist, medical oncologist, radiologist, and pathologist).

Inclusion criteria were as follows: (1) patients between 18 and 85 years of age, (2) histopathological confirmation of peritoneal metastases of gastric cancer, and (3) those who received a diagnostic laparoscopy at our department. The exclusion criteria were as follows: (1) patients with any other distant metastases (e.g., liver, lung, and brain), (2) those with missing PCI documentation, and (3) patients with regional cytotoxic treatment (e.g., pressurized intraperitoneal aerosol chemotherapy) during the follow-up period. The study was approved by the local ethics committee (EA1/009/16). The median follow-up was 7.3 months.

\section{Definition of the groups}

According to the recommendation of the MDT, patients were treated with either CRS (total or subtotal gastrectomy, a

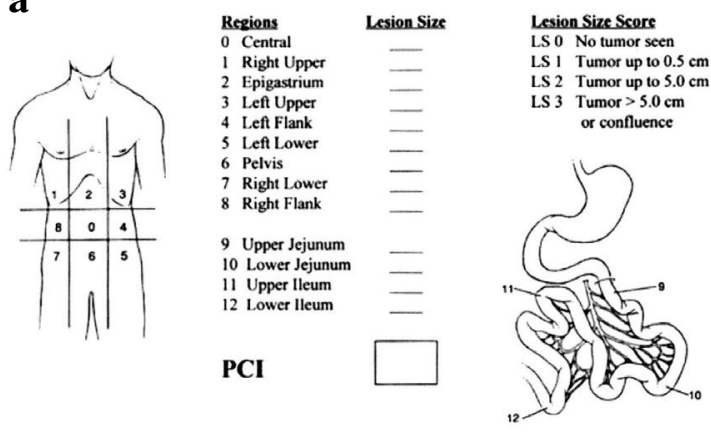

b

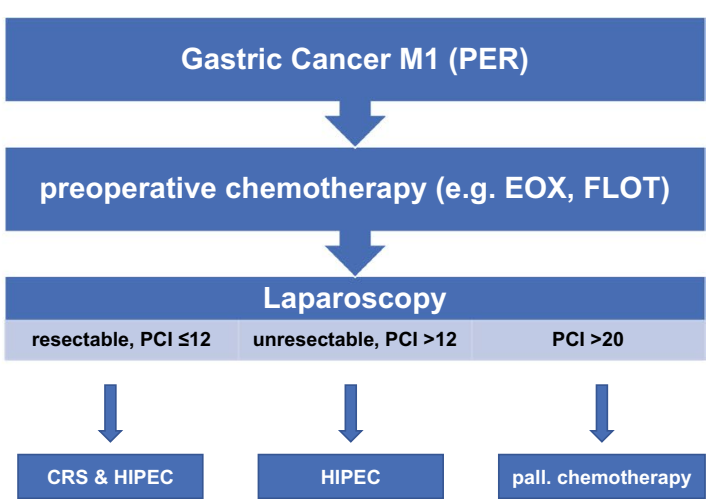

Fig. 1 a Peritoneal Cancer Index (PCI), with kind permission of Paul Sugarbaker [8]. b Flow chart illustrating decision about different treatment regimens for patient with gastric cancer and peritoneal metastasis 
omentectomy, peritonectomy, and additional organ resection if necessary) and HIPEC in combination with pre- and postoperative systemic chemotherapy in cases where complete cytoreduction seemed achievable (according to the PCI and the diagnostic laparoscopy). If complete cytoreduction did not seem achievable, systemic chemotherapy alone was administered to those with a high PCI (PCI > 20) and/ or unresectable peritoneal metastases. Peritoneal metastases were defined as unresectable if there was small bowel involvement in several locations. In case complete cytoreduction or gastrectomy was intraoperatively not achievable with tumor-free margins for patients with planned CRS and HIPEC, laparoscopy or laparotomy and HIPEC was performed with postoperative systemic chemotherapy. Patients received this form of treatment if they had a locally advanced primary tumor with infiltration in several organs, coeliac trunk, para-aortal lymph-node metastases, or unresectable peritoneal metastases (with no possibility of an R0 resection). Therefore, the three different treatment groups were defined as (I) CRS and HIPEC in combination with systemic chemotherapy (group 1); (II) laparoscopy/laparotomy and HIPEC in combination with systemic chemotherapy (group 2 ), and (III) systemic chemotherapy only (group 3). The treatment scheme is shown in Fig. 1b. The final PCI was evaluated based on the explorative laparotomy for group 1 and 2 group and by diagnostic laparoscopy for group 3 .

\section{Treatment of the different groups}

According to the German national S3-guidelines and NCNN guidelines for stage IV gastric cancer, the majority of patients were initially treated with systemic chemotherapy including a platin/5-FU-based combination of chemotherapy (e.g., oxaliplatin, cisplatin in combination with 5-FU, leucovorin, or docetaxel).

CRS was carried out to achieve complete cytoreduction. For this treatment, patients underwent total or subtotal gastrectomy with Roux-Y reconstruction. Peritonectomy procedures included the diaphragmatic peritonectomy, parietal peritonectomy, and pelvic peritonectomy in those with peritoneal metastases in these quadrants. Patients also received an omentectomy. Multi-visceral resection was defined as a partial resection of liver, pancreas, small bowel or colon; splenectomy, cholecystectomy, and ovarectomy were not defined as multi-visceral resection. Complete cytoreduction was defined as tumor nodules less than $2.5 \mathrm{~mm}$ in size $(\mathrm{CC}=1)$ or the absence of visible tumor nodules $(\mathrm{CC}=0)$. Patients who were treated with CRS or laparotomy/laparoscopy received immediate HIPEC (cisplatin $75 \mathrm{mg} / \mathrm{m}^{2}$, mitomycin $15 \mathrm{mg} / \mathrm{m}^{2}, 60 \mathrm{~min}$, and mean temperature $41{ }^{\circ} \mathrm{C}$ ) distributed in $62(89.9 \%)$ patients through a closed circulatory technique and in $7(10.1 \%)$ patients through an open circulatory technique. The postoperative chemotherapy was mainly platin/5-FU-based and was changed to the secondline chemotherapy, including docetaxel or irinotecan in those with tumor recurrence or progress.

\section{Clinical data, survival, and follow-up}

Clinical data were collected during regular follow-up visits either in the surgical or oncological department and no patient was lost to follow-up.

Overall survival was calculated from the day of CRS or laparotomy/laparoscopy and HIPEC for patients of groups 1 and 2 and from the day of diagnostic laparoscopy for group 3 until the patient's death or last contact.

\section{Histopathologic data}

Histopathologic investigation was performed by an experienced pathologist in every case. Tumor regression scores were calculated according to the classification of Becker [15].

\section{Statistical analysis}

All statistical analyses performed were exploratory in nature and performed using either SPSS 23.0 (International Business Machines Corporation, Armonk, NY, USA) or Prism 6.0 (Graphpad Software, Inc., La Jolla, CA, USA). Continuous descriptive data are given as mean and standard deviation. Categorical data are given as frequencies and proportions. Univariate analysis of time to event data was performed using log-rank test, including $25 \%$ and $75 \%$ quartiles to compare several groups. Univariate results were visualized by Kaplan-Meier curves. To identify independent risk factors and protective factors in addition to group comparisons, a multiple Cox-proportional hazard regression analysis was applied. All variables, which showed a $p$ value below 0.10 in univariate analysis, were considered for the Cox-regression model. Backward stepwise variable selection with the Akaike information criterion (AIC) method was used. A $p$ value below 0.05 was considered significant.

\section{Results}

A total of 88 patients with a mean age of $54.6 \pm 10.9$ years, a mean BMI of $24.4 \pm 4.7$, and a mean PCI of $14.3 \pm 11.3$ were included. In total, 50 patients were female, 83 patients were preoperatively treated with chemotherapy, and all had synchronous peritoneal metastases. The mean time from diagnosis to laparoscopy was $5.2 \pm 2.9$ months. Group 1 contained 58 patients, group 2 contained 11 patients, and group 3 contained 19 patients. 
The main demographic difference between the three groups was the PCI $(p<0.001)$, and cardiac comorbidities $(p=0.02)$. The demographic data of the full cohort and the groups are shown in Table 1.

\section{Oncologic treatment}

In total, 83 (94.3\%) patients received a median of 3 (1-16) cycles of preoperative chemotherapy. The most commonly

Table 1 Patient demographics and chemotherapy regimen from the three different treatment groups

\begin{tabular}{|c|c|c|c|c|c|}
\hline Factor & Total $(n=88)$ & Group $1(n=58)$ & Group $2(n=11)$ & Group $3(n=19)$ & $p$ value \\
\hline Female $(\%)$ & $55.8(50 / 88)$ & $55.2(32 / 58)$ & $54.5(6 / 11)$ & $63.2(12 / 19)$ & 0.82 \\
\hline Age (years) & $54.6 \pm 10.9$ & $53.9 \pm 10.2$ & $52.9 \pm 13.9$ & $57.8 \pm 11.1$ & 0.35 \\
\hline BMI $\left(\mathrm{kg} / \mathrm{m}^{2}\right)$ & $24.4 \pm 4.7$ & $24.5 \pm 5.1$ & $24.5 \pm 4.0$ & $24.0 \pm 3.6$ & 0.95 \\
\hline $\begin{array}{l}\text { Time from diagnosis to HIPEC/lapa- } \\
\text { roscopy (months) }\end{array}$ & $5.2 \pm 2.9$ & $5.5 \pm 3.2$ & $5.2 \pm 1.9$ & $4.2 \pm 2.2$ & 0.21 \\
\hline PCI Score & $14.3 \pm 11.3$ & $8.3 \pm 5.7$ & $23.9 \pm 11.1$ & $27.3 \pm 9.3$ & $<0.001$ \\
\hline Her-2-neu status pos. (\%) & $20.5(15 / 73)$ & $19.6(10 / 51)$ & $33.3(3 / 9)$ & $15.4(2 / 13)$ & 0.59 \\
\hline n.a. & $17.0(15 / 88)$ & $12.1(7 / 58)$ & $18.2(2 / 11)$ & $31.6(6 / 19)$ & \\
\hline Signet ring cell carcinoma $(\%)$ & $61.6(53 / 86)$ & $65.5(38 / 58)$ & $54.5(6 / 11)$ & $52.9(9 / 17)$ & 0.55 \\
\hline n.a. & $2.3(2 / 88)$ & & & $10.5(2 / 19)$ & \\
\hline Tumor localization (\%) & & & & & 0.48 \\
\hline Cardia & $14.9(13 / 87)$ & $15.5(9 / 58)$ & 0 & $22.2(4 / 18)$ & \\
\hline Fundus & $3.4(3 / 87)$ & $5.2(3 / 58)$ & 0 & 0 & \\
\hline Corpus & $43.7(38 / 87)$ & $43.1(25 / 58)$ & $45.5(5 / 11)$ & $44.4(8 / 18)$ & \\
\hline Antrum & $31.0(27 / 87)$ & $29.3(17 / 58)$ & $54.5(6 / 11)$ & $22.2(4 / 18)$ & \\
\hline Dysplastica & $6.9(6 / 87)$ & $6.9(4 / 58)$ & 0 & $11.1(2 / 18)$ & \\
\hline n.a. & $1.1(1 / 88)$ & & & $5.3(1 / 19)$ & \\
\hline \multicolumn{6}{|l|}{ Comorbidities (\%) } \\
\hline None & $47.7(42 / 88)$ & $46.6(27 / 58)$ & $72.7(8 / 11)$ & $36.8(7 / 19)$ & 0.16 \\
\hline Pulmonal & $5.7(5 / 88)$ & $8.6(5 / 58)$ & 0 & 0 & 0.25 \\
\hline Cardiac & $13.6(12 / 88)$ & $10.3(6 / 58)$ & 0 & $31.6(6 / 19)$ & 0.02 \\
\hline Renal & $1.1(1 / 88)$ & $1.7(1 / 58)$ & 0 & 0 & 0.77 \\
\hline Metabolic & $21.6(19 / 88)$ & $17.2(10 / 58)$ & $18.2(2 / 11)$ & $36.8(7 / 19)$ & 0.19 \\
\hline Vascular & $4.5(4 / 88)$ & $6.9(4 / 58)$ & 0 & 0 & 0.34 \\
\hline Liver & $3.4(3 / 88)$ & $3.4(2 / 58)$ & 0 & $5.3(1 / 19)$ & 0.75 \\
\hline ASA Score (\%) & & & & & 0.33 \\
\hline I & $4.8(4 / 84)$ & $3.4(2 / 58)$ & 0 & $13.3(2 / 15)$ & \\
\hline II & $47.6(40 / 84)$ & $53.4(31 / 58)$ & $36.4(4 / 11)$ & $33.3(5 / 15)$ & \\
\hline III & $45.2(38 / 84)$ & $41.4(24 / 58)$ & $63.6(7 / 11)$ & $46.7(7 / 15)$ & \\
\hline IV & $2.4(2 / 84)$ & $1.7(1 / 58)$ & 0 & $6.7(1 / 15)$ & \\
\hline n.a. & $4.5(4 / 88)$ & $(/ 58)$ & $(/ 11)$ & $21.1(4 / 19)$ & \\
\hline Preoperative chemo (\%) & $94.3(83 / 88)$ & $98.3(57 / 58)$ & $100(11 / 11)$ & $78.9(15 / 19)$ & 0.005 \\
\hline Number of cycles (median) & $3(1-16)$ & $4(1-12)$ & $3(1-4)$ & $4(1-16)$ & 0.35 \\
\hline n.a. & $15.9(14 / 88)$ & $6.9(4 / 58)$ & $36.4(4 / 11)$ & $31.6(6 / 19)$ & \\
\hline Chemotherapeutic regimens (\%) & & & & & 0.28 \\
\hline FLOT & $55.4(46 / 83)$ & $61.4(35 / 57)$ & $36.4(4 / 11)$ & $46.7(7 / 15)$ & \\
\hline ECF/ECX & $28.9(24 / 83)$ & $22.8(13 / 57)$ & $54.5(6 / 11)$ & $33.3(5 / 15)$ & \\
\hline Others & $15.7(13 / 83)$ & $15.8(9 / 57)$ & $9.1(1 / 11)$ & $20(3 / 15)$ & \\
\hline Postoperative chemo (\%) & $77.4(48 / 62)$ & $71.4(25 / 35)$ & $77.8(7 / 9)$ & $88.9(16 / 18)$ & 0.36 \\
\hline n.a. & $29.5(26 / 88)$ & $39.7(23 / 58)$ & $18.2(2 / 11)$ & $5.3(1 / 19)$ & \\
\hline Number of cycles (median) & $3.3 \pm 1.6$ & $3.4 \pm 1.0$ & $4.0 \pm 2.9$ & $2.8 \pm 2.2$ & 0.45 \\
\hline n.a. & $62.5(55 / 88)$ & $65.5(38 / 58)$ & $63.6(7 / 11)$ & $52.6(10 / 19)$ & \\
\hline
\end{tabular}

Group 1 CRS and HIPEC, Group 2 laparoscopy/laparotomy and HIPEC, Group 3 systemic chemotherapy only, PCI Peritoneal Cancer Index, n.a. not available (continuous data are shown as mean and standard deviations), ASA Score American Society of Anesthesiologists Score, FLOT docetaxel, oxaliplatin, and fluorouracil/leucovorin, ECF/ECX epirubicin, cisplatin, and fluorouracil or capecitabine 
used chemotherapeutic regimes were docetaxel, oxaliplatin, and fluorouracil/leucovorin (FLOT) in $46(55.4 \%)$ of all patients and epirubicin, cisplatin, and fluorouracil or capecitabine (ECF/ECX) in 24 (28.9\%) of all patients. Group 3 contained significantly more patients without preoperative chemotherapy ( $4 / 19$ vs. $1 / 58$ vs. $0 / 11 ; p=0.005)$. The number of cycles and chemotherapeutic regimens did not differ between the three different treatment groups (Table 1).

\section{Overall survival}

The median overall survival of patients was $9.8 \pm 0.7$ for group 1, $6.3 \pm 3.0$ for group 2 and $4.9 \pm 1.9$ for group 3 $(p<0.001)$. The 1 -year survival was $40.9 \%$ vs. $12.1 \%$ vs. $0 \%$, the 2 -year survival was $23.4 \%$ vs. $12.1 \%$ vs. $0 \%$, and the 3 -year survival was $17.5 \%$ vs. $0 \%$ vs. $0 \%$ for group 1 , 2, and 3, respectively (Fig. 2). Five-year patient survival could only be reached in group 1 and was $7.0 \%$. Univariate analysis of overall patient survival in all patients treated with CRS and HIPEC confirmed lymph-node metastasis, Peritoneal Cancer Index $\geq 12$, incomplete cytoreduction, margin positive resection (R1/2), multi-visceral resection, and $>4$ cycles of preoperative chemotherapy as factors which

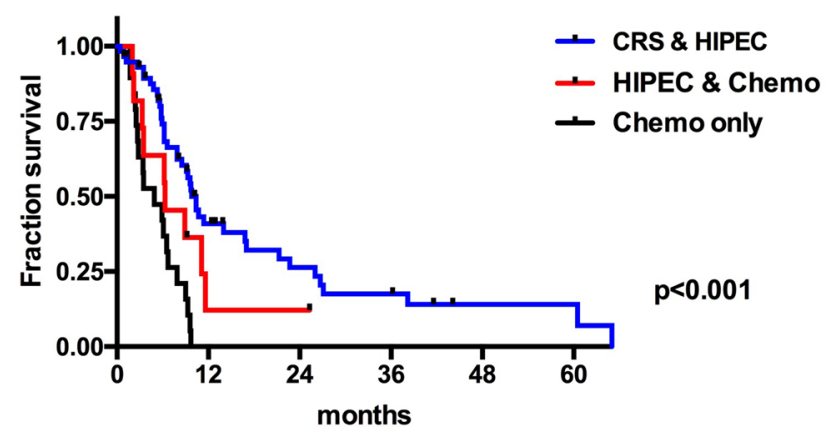

Fig. 2 Overall patient survival comparing patients of the three treatment groups

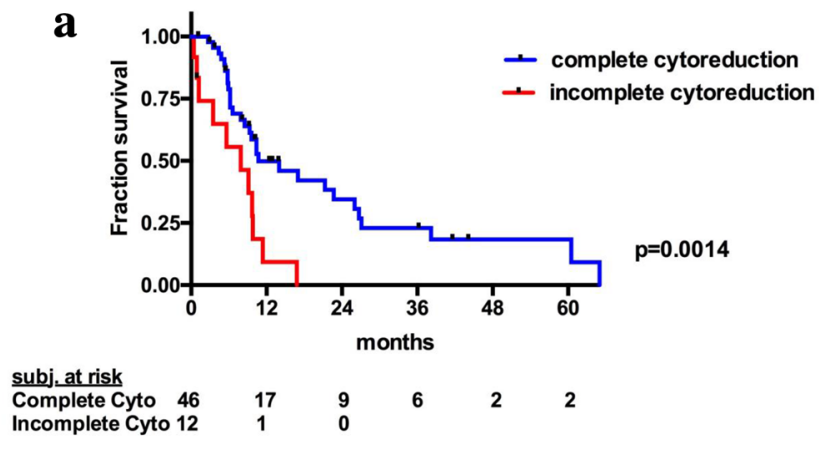

Fig. 3 Overall patient survival comparing patients according to the completeness of cytoreduction. a Patients with or without complete cytoreduction treated with cytoreductive surgery and hyperthermic impaired overall patient survival. Other factors including gender, age $\geq 60$ years, tumor location (cardia/fundus vs. corpus/antrum), depth of invasion, HER2-status positivity, signet ring cell carcinoma, tumor regression, ASA Score, preoperative chemotherapy regime (FLOT vs. ECF/ECX), and peritoneal dissemination (region 0-4 vs. region 5-13) were not significantly associated with patient survival (logrank test $=0.81 ; 0.85 ; 0.20 ; 0.45 ; 0.16 ; 0.054 ; 0.15 ; 0.058$; $0.77 ; 0.68)$. Those with tumor regression of grade 1 according to Becker showed better median survival compared to grade 2 and 3 (60.5 vs. 9.8 months, respectively), but did not reach significance ( $n=10$ vs. 47 , respectively, $p=0.15$ ). Incomplete cytoreduction and laparotomy/laparoscopy in combination with HIPEC showed no improvement on overall survival compared to the group $3(p=0.08)$ (Fig. 3). A subgroup analysis of patients treated with CRS and HIPEC and $\mathrm{PCI}<12$ proved the significance while comparing patients with $\leq 4$ cycles of preoperative chemotherapy compared to patients with $>4$ cycles (median survival 22.7 vs. 8.5 months; $p<0.001)$. Patients treated with CRS and HIPEC and only limited peritoneal disease $(\mathrm{PCI}<6)$ showed a postoperative overall survival of 17.0 months, resulting in a survival from diagnosis to last follow-up of 24.9 months.

Cox-regression analysis revealed four predictive factors associated with impaired patient survival: lymphnode metastasis (HR 3.53, $p=0.005$ ), $\mathrm{PCI} \geq 12$ (HR 2.11, $p=0.036$ ), incomplete cytoreduction (HR 4.30, $p=0.001$ ), and $>4$ cycles of preoperative chemotherapy (HR 4.49 , $p<0.001$ ) (Table 2; Fig. 4).

\section{Preoperative chemotherapy}

Focusing the analysis on preoperative intravenous chemotherapy, we divided group 1 in patients who received $\leq 4$ cycles of preoperative intravenous chemotherapy $(n=37)$ and patients who received $>4$ cycles of preoperative chemotherapy $(n=15)$. The analysis of patient and tumor

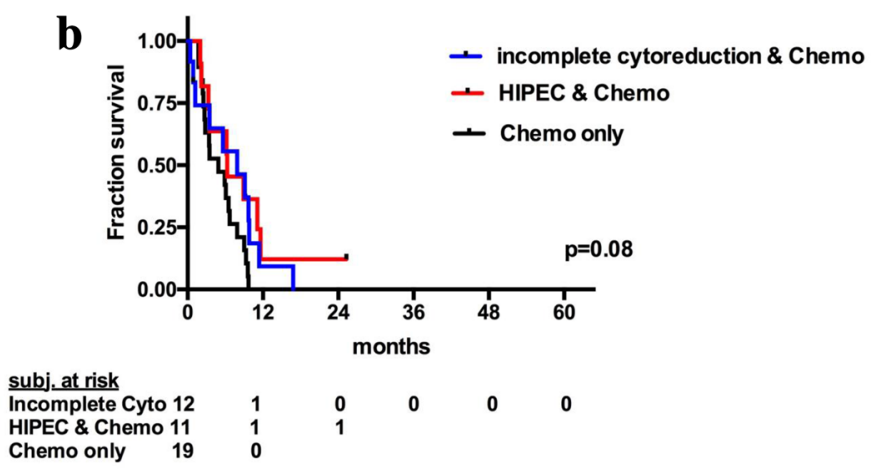

intraperitoneal chemotherapy. b Patients with incomplete cytoreduction or laparotomy combined with hyperthermic intraperitoneal chemotherapy compared to intravenous chemotherapy 
Table 2 Uni- and multi-variate analyses of factors affecting survival among gastric cancer patients with peritoneal metastasis after cytoreductive surgery and hyperthermic intraperitoneal chemotherapy

\begin{tabular}{|c|c|c|c|c|c|c|c|}
\hline \multirow[t]{2}{*}{ Variable } & \multirow[t]{2}{*}{ Category } & \multirow[t]{2}{*}{$N$} & \multirow{2}{*}{$\begin{array}{l}\text { Median overall } \\
\text { survival (months) }\end{array}$} & \multirow{2}{*}{$\begin{array}{l}\text { Univariate analy- } \\
\text { sis ( } p \text { value })\end{array}$} & \multicolumn{3}{|c|}{ Multi-variate analysis } \\
\hline & & & & & HR & $95 \% \mathrm{CI}$ & $p$ value \\
\hline \multirow[t]{2}{*}{ Gender } & Male & 26 & $10.4(6.2-17.0)$ & 0.81 & & & \\
\hline & Female & 32 & $9.7(5.8-27.1)$ & & & & \\
\hline \multirow[t]{2}{*}{ Age } & $<60$ years & 38 & $9.6(5.9-17.0)$ & 0.85 & & & \\
\hline & $\geq 60$ years & 20 & $14.0(5.8-27.1)$ & & & & \\
\hline \multirow[t]{2}{*}{ Tumor location } & Proximal (cardia, fundus) & 17 & $10.7(6.3-12.4)$ & 0.20 & & & \\
\hline & Distal (corpus/antrum) & 47 & $9.3(5.8-22.7)$ & & & & \\
\hline \multirow[t]{2}{*}{ Depth of invasion } & Т $0-3$ & 25 & $11.4(7.9-26.7)$ & 0.45 & & & \\
\hline & T 4 & 33 & $9.7(5.8-17.0)$ & & & & \\
\hline \multirow[t]{2}{*}{ Lymph-node metastasis } & $\mathrm{N} 0$ & 12 & $26.7(5.0-48.4)$ & 0.034 & 1 & $1.46-8.57$ & 0.005 \\
\hline & $\mathrm{N} 1-3$ & 46 & $9.3(5.8-17.0)$ & & 3.53 & & \\
\hline \multirow[t]{2}{*}{ Her-2 } & Negative & 41 & $9.6(8.1-11.1)$ & 0.29 & & & \\
\hline & Positive & 10 & $17.0(2.4-31.6)$ & & & & \\
\hline \multirow[t]{2}{*}{ Histology } & Adenocarcinoma & 20 & $22.7(6.2-38.2)$ & 0.054 & 1 & $0.61-3.72$ & 0.373 \\
\hline & Signet ring cell carcinoma & 38 & $9.6(5.8-17.0)$ & & 1.51 & & \\
\hline \multirow[t]{2}{*}{ Tumor regression } & Becker grade 1 & 10 & 60.5 & 0.15 & 1 & $1.05-4.25$ & $\mathbf{0 . 0 3 6}$ \\
\hline & Becker grade $2+3$ & 47 & $9.8(8.5-11.1)$ & & 2.11 & & \\
\hline \multirow[t]{2}{*}{ Peritoneal Cancer Index } & $<12$ & 38 & $14(6.6-26.7)$ & $\mathbf{0 . 0 3 3}$ & 1 & $1.05-4.25$ & $\mathbf{0 . 0 3 6}$ \\
\hline & $\geq 12$ & 20 & $7.9(4.8-16.8)$ & & 2.11 & & \\
\hline \multirow[t]{2}{*}{ Cytoreduction } & Complete & 46 & $10.7(6.2-27.1)$ & 0.001 & 1 & $1.87-9.89$ & 0.001 \\
\hline & Incomplete & 12 & $7.9(1.2-9.8)$ & & 4.30 & & \\
\hline \multirow[t]{2}{*}{ Local tumor resection } & R 0 & 39 & $14(6.2-27.1)$ & 0.040 & 1 & $0.69-3.05$ & 0.320 \\
\hline & $\mathrm{R} 1 / 2$ & 19 & $7.9(3.5-11.4)$ & & 1.46 & & \\
\hline \multirow[t]{2}{*}{ ASA Score } & $1 / 2$ & 33 & $8.5(5.4-21.3)$ & 0.058 & 1.52 & $0.66-3.49$ & 0.321 \\
\hline & $3 / 4$ & 25 & $16.8(9.6-38.2)$ & & 1 & & \\
\hline \multirow[t]{2}{*}{ Resection } & Multi-visceral & 11 & $6.2(5.6-10.4)$ & 0.003 & 1.56 & $0.50-4.82$ & 0.445 \\
\hline & Gastrectomy & 47 & $14.0(6.2-27.1)$ & & 1 & & \\
\hline \multirow[t]{2}{*}{ Preoperative chemo } & FLOT & 36 & $9.7(5.8-27.1)$ & 0.77 & & & \\
\hline & $\mathrm{ECF} / \mathrm{ECX}$ & 13 & $10.4(6.2-22.7)$ & & & & \\
\hline \multirow[t]{2}{*}{ Preoperative chemotherapy } & $1-4$ cycles & 38 & $14(6.2-38.2)$ & 0.002 & 1 & $1.95-10.37$ & $<0.001$ \\
\hline & $>4$ cycles & 14 & $6.2(5.6-9.8)$ & & 4.49 & & \\
\hline \multirow[t]{2}{*}{ Peritoneal dissemination } & Region 0-4 & 19 & $11.4(8.5-21.3)$ & 0.68 & & & \\
\hline & Region 5-13 & 34 & $9.7(5.6-27.1)$ & & & & \\
\hline
\end{tabular}

$H R$ hazard ratio, $C I$ confidence interval; overall survival is illustrated as median with $25 \%$ and $75 \%$ quartiles, FLOT docetaxel, oxaliplatin, and fluorouracil/leucovorin, $E C F / E C X$ epirubicin, cisplatin, and fluorouracil or capecitabine

demography showed no significant difference, except the median number of preoperative chemotherapy cycles (4 vs. 6 cycles; $p<0.001$ ), and is illustrated in Table 3 .

Comparing the postoperative complication rates between these two groups, there was no significant difference in surgical or medical complication $(p=0.72)$ (data not shown). The median postoperative hospital stay and the median stay on the intensive care unit did not differ significantly (12.0 (8-375) vs. 13.5 (7-31) days; $p=0.39$ and $2.0(1-368)$ vs. $2.0(1-5)$ days; $p=0.33)$.

The median progression-free survival of patients treated with $\leq 4$ cycles of preoperative chemotherapy was higher compared to patients treated with $>4$ cycles of preoperative chemotherapy but missed the level of significance (12.9 vs. 5.4 months; $p=0.09$ ).

\section{Complication rate}

\section{Group 1}

Overall, 13 of 58 patients (22.4\%) developed Clavien/Dindo grade III/IV postoperative complications in the CRH group. The mortality rate was $1.7 \%$. This patient died after CRS and 
a

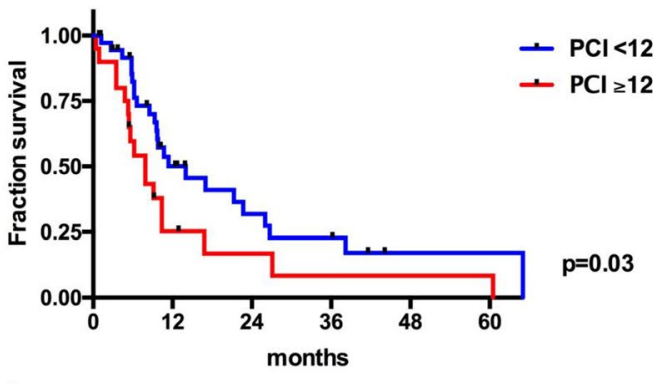

subj. at risk

$\mathrm{PCl}<12$

$\mathrm{PCl} \geq 12$

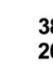

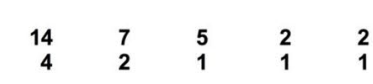

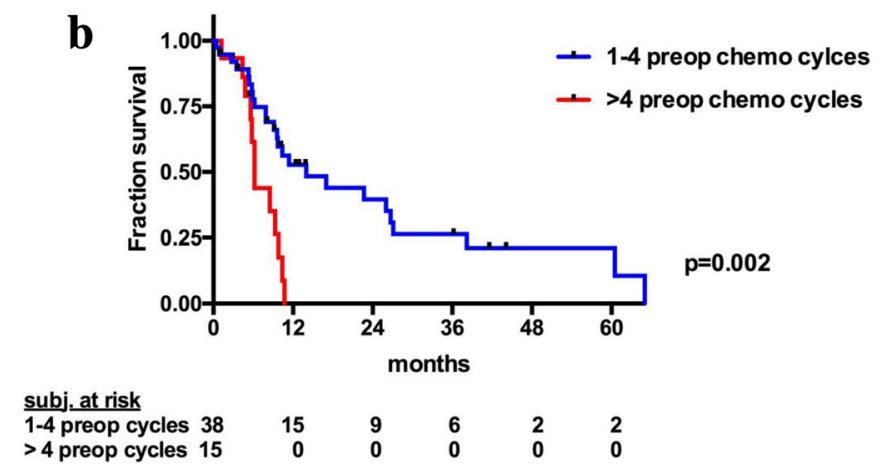

Fig. 4 Overall patient survival comparing different factors using logrank test. a Patients with Peritoneal Cancer Index (PCI) $<12$ or $\geq 12$ treated with cytoreductive surgery and hyperthermic intraperitoneal chemotherapy. b Patients treated with 1-4 or more than 4 chemotherapy cycles prior cytoreductive surgery and hyperthermic intraperitoneal chemotherapy
Table 3 Patient demographics and chemotherapy regimen from two different treatment groups according to the number of cycles of preoperative chemotherapy

\begin{tabular}{|c|c|c|c|c|}
\hline Factor & Total $(n=58)$ & $\leq 4$ cycles $(n=37)$ & $>4$ cycles $(n=15)$ & $p$ value \\
\hline Female $(\%)$ & $55.8(29 / 52)$ & $59.5(22 / 37)$ & $46.7(7 / 15)$ & 0.30 \\
\hline Age (years) & $54.0 \pm 10.7$ & $55.8 \pm 9.1$ & $49.5 \pm 13.2$ & 0.053 \\
\hline BMI $\left(\mathrm{kg} / \mathrm{m}^{2}\right)$ & $24.6 \pm 5.3$ & $24.9 \pm 5.3$ & $23.9 \pm 5.1$ & 0.56 \\
\hline PCI Score & $8.0 \pm 5.7$ & $8.2 \pm 5.6$ & $7.3 \pm 6.0$ & 0.58 \\
\hline Her-2-neu status pos. (\%) & $17.4(8 / 46)$ & $17.6(6 / 34)$ & $16.7(2 / 12)$ & 0.66 \\
\hline n.a. & $20.7(12 / 58)$ & $8.1(3 / 37)$ & $20(3 / 15)$ & \\
\hline Nodal positive (\%) & $76.9(40 / 52)$ & $78.4(29 / 37)$ & $73.3(11 / 15)$ & 0.48 \\
\hline Tumor regression Becker $1(\%)$ & $19.2(10 / 52)$ & $18.9(7 / 37)$ & $20(3 / 15)$ & 0.60 \\
\hline Signet ring cell carcinoma (\%) & $63.5(33 / 52)$ & $59.5(22 / 37)$ & $73.3(11 / 15)$ & 0.27 \\
\hline \multicolumn{5}{|l|}{ Comorbidities (\%) } \\
\hline None & $46.2(19 / 52)$ & $51.4(19 / 37)$ & $33.3(5 / 15)$ & 0.19 \\
\hline Pulmonal & $7.7(4 / 52)$ & $5.4(2 / 37)$ & $13.3(2 / 15)$ & 0.33 \\
\hline Cardiac & $11.5(6 / 52)$ & $10.8(4 / 37)$ & $13.3(2 / 15)$ & 0.57 \\
\hline Renal & $1.9(1 / 52)$ & $2.7(1 / 37)$ & 0 & 0.71 \\
\hline Metabolic & $15.4(8 / 52)$ & $16.2(6 / 37)$ & $13.3(2 / 15)$ & 0.58 \\
\hline Vascular & $7.7(4 / 52)$ & $8.1(3 / 37)$ & $6.7(1 / 15)$ & 0.67 \\
\hline Liver & $3.8(2 / 52)$ & $2.7(1 / 37)$ & $6.7(1 / 15)$ & 0.50 \\
\hline ASA Score $(\%)$ & & & & 0.23 \\
\hline I & $1.9(1 / 52)$ & 0 & $6.7(1 / 15)$ & \\
\hline II & $55.8(29 / 52)$ & $51.4(19 / 37)$ & $66.7(10 / 15)$ & \\
\hline III & $40.4(21 / 52)$ & $45.9(17 / 37)$ & $26.7(4 / 15)$ & \\
\hline IV & $1.9(1 / 52)$ & $2.7(1 / 37)$ & 0 & \\
\hline Complete cytoreduction (\%) & $78.8(41 / 52)$ & $78.4(29 / 37)$ & $80.0(12 / 15)$ & 0.61 \\
\hline Number of cycles (median) & $4(1-12)$ & $4(1-4)$ & $6(5-12)$ & $<0.001$ \\
\hline
\end{tabular}

n.a. not available (continuous data are shown as mean and standard deviations), ASA Score American Society of Anesthesiologists Score
HIPEC due to multiple small bowel perforations with sepsis and multi-organ-failure.

\section{Group 2}

None of the patients developed Clavien/Dindo grade III/ IV postoperative complications or died after laparotomy/ laparoscopy and HIPEC. Three of eleven patients (27.3\%) developed minor complications (Clavien/Dindo grade II) and were treated with antibiotics. 


\section{Discussion}

Our study demonstrated significantly improved overall patient survival in those with peritoneal metastases of gastric cancer treated with complete cytoreduction and HIPEC in the largest European study using a singlecenter design. Five-year survival could only be reached in patients treated with CRS and HIPEC and who had a PCI $<12$, highlighting the potential of this treatment option for patients with low PCI and achievable complete cytoreduction. One of the main strengths of this study is that every patient, even in group 3, was staged with diagnostic laparoscopy and PCI at our institute. This aspect allowed us to make a comparison between those with complete cytoreduction or laparotomy/laparoscopy in combination with HIPEC with those patients with peritoneal metastases as only metastatic site who were staged with laparoscopy and treated with systemic chemotherapy alone. The majority of studies regarding peritoneal metastases of gastric cancer derive from Asia. Imamoto et al. demonstrated an overall survival of patients with malignant ascites and gastric cancer with intravenous paclitaxel treatment administered for 5.2 months [16]. Moreover, the efficacy of cytoreduction and HIPEC after neoadjuvant intraperitoneal and systemic chemotherapy (NIPS) for patients with advanced peritoneal dissemination of gastric cancer and a median overall survival of 20.4 months compared to bidirectional chemotherapy only (10.5 months) have been shown by Yonemura et al. [17, 18]. In comparison, our study does not support a survival benefit for patients who received incomplete cytoreduction or laparoscopy/ laparotomy with HIPEC compared to patients treated with systemic chemotherapy alone, which is in line with the previously mentioned studies.

The results of this study confirmed the generally accepted approach of patient selection based on the PCI score and the patient's potential for complete cytoreduction. Although the patients in group 2 had a low complication rate, this therapeutic option showed better but not significant increase in overall survival in this small subseries of patients $(n=11)$ compared to group $3(n=19)$. The PCI in group 2 was comparable to group 3 (23.9 vs. 27.3), respectively. According to Badgwell et al., single or repeated laparoscopic HIPEC as pre-CRS treatment for patients with positive cytology and peritoneal metastases may help to reduce peritoneal metastases prior to gastrectomy and HIPEC [19]. Yonemura et al. investigated a series of patients treated with laparoscopic HIPEC in combination with NIPS prior to CRS and HIPEC. The laparoscopy was beneficial for reevaluating the peritoneal metastases during the NIPS treatment for patient selection [20]. According to the literature, it is not surprising that group 2 with a single HIPEC procedure could not significantly influence patient survival in our study. In contrast to previously mentioned studies, group 2 consisted of patients with a high PCI. There might be a hypothetical benefit in regional tumor control of peritoneal metastases for repeated applications in a palliative setting. Regarding the most recent development of pressurized intraperitoneal aerosol chemotherapy (PIPAC) with low-dose cisplatin and doxorubicin and its demonstrated tumor response rate of up to $50 \%$ in combination with a low complication rate, repeated laparoscopic HIPEC might not play a role in cancer treatment alone [21].

\section{Predictive factors on overall patient survival}

\section{Completeness of cytoreduction}

Completeness of cytoreduction is the most important predictive factor for overall survival in patients with peritoneal metastases of gastric cancer. The two largest studies from Canbay et al. and Glehen et al. focusing on multimodal treatment including CRS and HIPEC for peritoneal metastases in patients with gastric cancer demonstrated the importance of complete cytoreduction and PCI [9, 22]. Our study and its results are in line with these important finding.

\section{Lymph-node metastasis}

There is limited evidence about the impact of lymph-node metastasis in patients with peritoneal metastasis of gastric cancer. The pathway of metastasizing is not very well understood. There are mainly two theories of metastasizing to the peritoneum: (I) growth through the serosal layer of the stomach and (II) tumor cell migration through the lymph nodes. The latter one is assumed for patients with the early stage of gastric cancer, limited to the mucosa or submucosa who developed lymph-node metastases and peritoneal metastases [23]. Lymph-node metastases are a well-known negative prognostic factor in curative treatment for gastric cancer. It impairs overall survival and increases tumor recurrence $[4,24]$. Due to the fact of limited evidence for the absence of lymph-node metastasis in patients with gastric cancer and peritoneal metastasis, it has been shown that patients with distant metastasis of colorectal cancer which spared the lymph nodes had a better prognosis [25]. Taking into consideration that the lack of lymph-node metastasis is associated with a minor systemic component of the cancer disease, with its serosal migration and regional tumor spread, these patients might have a less malignant tumor biology and, therefore, benefit from CRS and HIPEC. 


\section{Preoperative chemotherapy}

Our study was able to demonstrate a potential negative effect of prolonged ( $>4$ cycles) preoperative intravenous chemotherapy on overall survival in patients qualified for CRS and HIPEC. Patients with PCI $<12$ showed significant reduced overall survival while treated with $>4$ cycles of preoperative chemotherapy.

In general, there are several potential explanations for this effect: (early) chemo-resistance, associated higher perioperative morbidity and mortality, selection bias, or center bias.

Chemotherapy resistance in patients with gastric cancer is a well-known fact which hampers the effect of intensive chemotherapy. Multi-drug resistance refers to multifactor and complex networks and is described in two forms: intrinsic or acquired. The major mechanism incorporate increasing drug efflux, decreasing drug influx, altering apoptotic machinery, altering the cell cycle checkpoints, enhancing drug metabolism, increasing DNA repair mechanisms, altering molecular drug targets, etc. [26-28]. A study by Ji et al. described that mesenchymal stem cells are implicated in the potentiation of drug resistance in gastric cancer and may be induced by extracellular vesicles of mesenchymal stem cells activating the CaM-Ks/Raf/Mek/ERK signaling pathway [29].

We could demonstrate that the pathologic regression rate of the primary was not significantly different between both groups (Becker Grade 1: $18.9 \%$ vs. $20 \% ; p=0.60$ ). The progression-free survival showed a difference, but did not reach level of significance (12.9 vs. 5.4 months; $p=0.09$ ) and might potentially support the hypothesis of less chemotherapeutic effect after four cycles.

Patients treated with $>4$ cycles of preoperative chemotherapy did not show higher morbidity or mortality in our study.

The selection and center bias might play an important role in patient selection as patients who received more than four cycles of preoperative chemotherapy were potentially referred at a later time after diagnosis. The majority of these patients were initially treated at smaller oncologic centers.

Focusing on the number of preoperative chemotherapeutic cycles, Yonemura et al. demonstrated the preoperative application of three cycles of NIPS in patients with gastric cancer and peritoneal metastasis [22]. A recent publication proved efficacy and safety of systemic and intraperitoneal preoperative S1, cisplatin, and docetaxel in patients with marginally resectable gastric cancer using 2-4 cycles of chemotherapy [30]. It seems effective to perform a limited number of preoperative cycles of chemotherapy to have a maximum of response and not to face chemo-resistance.

There are limited data about the effect of preoperative chemotherapy in patients with peritoneal metastases of gastric cancer. A recent publication of Al-Batran et al. evaluated the impact of 4 cycles of neoadjuvant FLOT in patients with no, limited, or extensive metastatic disease. The authors could demonstrate an overall response rate according to RECIST in $36 / 60(60 \%)$ of the patients with limited metastatic disease and 55/127 (43\%) with extensive metastatic disease resulting in a median overall survival of 22.9 and 10.7 months, respectively [31]. The subgroup analysis of surgically treated patients within the group of limited disease showed a median survival of 31.3 months. These data are difficult to compare with our cohort due to the fact that this group contained only $2 / 18$ patients with limited peritoneal metastatic disease. Patients with limited peritoneal disease $(\mathrm{PCI}<6)$ of our presented cohort treated with CRS and HIPEC reached an overall median survival (date of diagnosis - last follow-up) of 24.9 months and are, hence, within the same range.

Tumor response in gastric cancer is an important predictive factor [15, 32]. Tumor regression has been proven to have major impact on overall survival in patients with advanced gastric cancer and neoadjuvant treatment and also peritoneal metastases of gastric cancer and NIPS [22, 33]. Our subgroup analysis of tumor regression in response to preoperative systemic chemotherapy missed the level of significance, but could clearly show a tendency towards this important predictor for survival. Especially for patients with peritoneal metastases of gastric cancer, this predictor could play an important role in patient selection. We have seen high PCI patients with good tumor response to preoperative intraperitoneal chemotherapy (NIPS), and therefore, those with a favorable tumor biology could benefit from CRS and HIPEC for improving survival.

\section{Effect of HIPEC}

The results of our study suggest that HIPEC is only beneficial for patients with complete cytoreduction. We could demonstrate that the overall survival of patients without resection of the primary tumor, who were treated with HIPEC, did not significantly differ from overall survival of patients treated with systemic chemotherapy only $(p=0.08)$. This effect might be easily explained by pharmacologic studies demonstrating a penetration depth of approx. $1.5 \mathrm{~mm}$ in a rodent HIPEC model using Cisplatin [34]. The evidence of the beneficial effect of HIPEC on patients with peritoneal metastases of gastric cancer is limited as many large cohort studies CRS and HIPEC are compared to palliative treatment than separating the treatment regime of CRS from HIPEC. There is only one randomized-controlled trial by Yang et al. which compared 68 Chinese patients treated with CRS vs. CRS and HIPEC. The study population contained a relatively high number of patients with PCI $>20(34 \%)$ compared to other major publications. Patients treated with CRS and HIPEC showed significantly longer median overall 
survival compared with patients treated with CRS (11.0 vs. 6.5 months; $p=0.045$ ) [35]. To answer this question in the European population, the ongoing German GASTRIPEC trial is recruiting patients and randomizing their treatment of CRS vs. CRS and HIPEC after 4 cycles of preoperative chemotherapy [36].

The major limitation of this study is that it only included patients from a single institution, where patients were selected, and therapeutic options recommended on the basis of our 20-year experience in peritoneal surface malignancies. Therefore, selection bias was inevitably a factor in patient enrollment. The main factors in patient selection were performance status, PCI, tumor biology and tumor response after preoperative chemotherapy. Therefore, a direct comparison of treatment options in patients with a $\mathrm{PCI}<12$ was not possible, due to the different group size. In total, 38 patients fulfilled this criterion in the group 1, while only one patient in group 2 and one patient in the group 3 showed $\mathrm{PCI} \leq 12$. One-year survival was $50.1 \%$ vs. $0 \%$ vs. $0 \%(p=0.02)$. Regarding the literature, there are no stratified survival data about systemic chemotherapy in low or high PCI patients, so that the question will stay unanswered to which extent patient selection itself might be the reason for better overall survival. Comparing our results to the Japanese experience using the NIPS treatment regime, Yonemura et al. demonstrated median survival of 9.6 months in 31 patients with P3 grade of dissemination (high PCI) and a median of 3.8 cycles of NIPS, which were not eligible for CRS and HIPEC due to progressive disease or partial response [17]. These results have to be carefully interpreted, as it is known that tumor genetics differ between the Japanese and Western population. For example, Sunakawa et al. demonstrated an association of variants in genes encoding for macrophage-related functions with clinical outcome in patients with locoregional gastric cancer and suggested that the genetic predisposition of the host may dictate the immune-related component of the tumor for progression in gastric cancer [37]. Furthermore, the parameter lymphnode involvement seems to differ between the Japanese and western population, which results in higher rates of lymph-node metastasis in non-Asian Americans compared to Asian Americans according to a retrospective analysis of 104 patients with early gastric cancer [38].

\section{Conclusion}

CRS and HIPEC showed convincing results in selected patients with peritoneal metastases of gastric cancer in whom CRS could achieve complete cytoreduction. Best results could be achieved in patients with low $\mathrm{PCI}(\mathrm{PCI}<6)$ leading to a median overall survival of 17.0 months after CRS and HIPEC. Prolonged duration of preoperative intravenous chemotherapy ( $<4$ cycles) seemed to reduce patient survival in patients suitable for CRS and HIPEC. Therefore, an early evaluation of the profitableness of CRS and HIPEC seems essential in patients with gastric cancer and peritoneal metastasis.

\section{Compliance with ethical standards}

Conflict of interest The authors declare that they have no conflict of interest.

Ethical standards All procedures followed were in accordance with the ethical standards of the responsible committee on human experimentation (institutional and national) and with the Helsinki Declaration of 1964 and later versions.

Informed consent Informed consent or substitute for it was obtained from all patients included in the study.

\section{References}

1. Gretschel S, Siegel R, Estevez-Schwarz L, Hunerbein M, Schneider U, Schlag PM. Surgical strategies for gastric cancer with synchronous peritoneal carcinomatosis. Br J Surg. 2006;93(12):1530 5. https://doi.org/10.1002/bjs.5513.

2. Chu DZ, Lang NP, Thompson C, Osteen PK, Westbrook KC. Peritoneal carcinomatosis in nongynecologic malignancy. A prospective study of prognostic factors. Cancer. 1989;63(2):364-7.

3. Esaki Y, Hirayama R, Hirokawa K. A comparison of patterns of metastasis in gastric cancer by histologic type and age. Cancer. 1990;65(9):2086-90.

4. Seyfried F, von Rahden BH, Miras AD, Gasser M, Maeder U, Kunzmann V, et al. Incidence, time course and independent risk factors for metachronous peritoneal carcinomatosis of gastric origin-a longitudinal experience from a prospectively collected database of 1108 patients. BMC Cancer. 2015;15:73. https://doi. org/10.1186/s12885-015-1081-8.

5. Ajani JA, Bentrem DJ, Besh S, D'Amico TA, Das P, Denlinger C, et al. Gastric cancer, version 2.2013: featured updates to the NCCN Guidelines. J Natl Compr Cancer Netw. 2013;11(5):531-46.

6. Glehen O, Gilly FN, Boutitie F, Bereder JM, Quenet F, Sideris $\mathrm{L}$, et al. Toward curative treatment of peritoneal carcinomatosis from nonovarian origin by cytoreductive surgery combined with perioperative intraperitoneal chemotherapy: a multi-institutional study of 1,290 patients. Cancer. 2010;116(24):5608-18. https:// doi.org/10.1002/cncr.25356.

7. Yonemura Y, Elnemr A, Endou Y, Hirano M, Mizumoto A, Takao $\mathrm{N}$, et al. Multidisciplinary therapy for treatment of patients with peritoneal carcinomatosis from gastric cancer. World J Gastrointest Oncol. 2010;2(2):85-97. https://doi.org/10.4251/wjgo. v2.i2.85.

8. Jacquet $\mathrm{P}$, Sugarbaker PH. Clinical research methodologies in diagnosis and staging of patients with peritoneal carcinomatosis. Cancer Treat Res. 1996;82:359-74.

9. Glehen O, Gilly FN, Arvieux C, Cotte E, Boutitie F, Mansvelt $\mathrm{B}$, et al. Peritoneal carcinomatosis from gastric cancer: a multiinstitutional study of 159 patients treated by cytoreductive surgery combined with perioperative intraperitoneal chemotherapy. Ann 
Surg Oncol. 2010;17(9):2370-7. https://doi.org/10.1245/s1043 4-010-1039-7.

10. Al-Batran SE, Hartmann JT, Probst S, Schmalenberg H, Hollerbach S, Hofheinz R, et al. Phase III trial in metastatic gastroesophageal adenocarcinoma with fluorouracil, leucovorin plus either oxaliplatin or cisplatin: a study of the Arbeitsgemeinschaft Internistische Onkologie. J Clin Oncol. 2008;26(9):1435-42. https ://doi.org/10.1200/JCO.2007.13.9378.

11. Bang YJ, Van Cutsem E, Feyereislova A, Chung HC, Shen L, Sawaki A, et al. Trastuzumab in combination with chemotherapy versus chemotherapy alone for treatment of HER2-positive advanced gastric or gastro-oesophageal junction cancer (ToGA): a phase 3, open-label, randomised controlled trial. Lancet. 2010;376(9742):687-97. https://doi.org/10.1016/S0140 $-6736(10) 61121-X$

12. Al-Batran SE, Hofheinz RD, Pauligk C, Kopp HG, Haag GM, Luley $\mathrm{KB}$, et al. Histopathological regression after neoadjuvant docetaxel, oxaliplatin, fluorouracil, and leucovorin versus epirubicin, cisplatin, and fluorouracil or capecitabine in patients with resectable gastric or gastro-oesophageal junction adenocarcinoma (FLOT4-AIO): results from the phase 2 part of a multicentre, open-label, randomised phase $2 / 3$ trial. Lancet Oncol. 2016;17(12):1697-708. https://doi.org/10.1016/s1470 -2045(16)30531-9.

13. Ychou M, Boige V, Pignon JP, Conroy T, Bouche O, Lebreton $\mathrm{G}$, et al. Perioperative chemotherapy compared with surgery alone for resectable gastroesophageal adenocarcinoma: an FNCLCC and FFCD multicenter phase III trial. J Clin Oncol. 2011;29(13):1715-21. https://doi.org/10.1200/jco.2010.33.0597.

14. Cunningham D, Allum WH, Stenning SP, Thompson JN, Van de Velde CJ, Nicolson M, et al. Perioperative chemotherapy versus surgery alone for resectable gastroesophageal cancer. N Engl J Med. 2006;355(1):11-20. https://doi.org/10.1056/nejmoa055531.

15. Becker K, Mueller JD, Schulmacher C, Ott K, Fink U, Busch $\mathrm{R}$, et al. Histomorphology and grading of regression in gastric carcinoma treated with neoadjuvant chemotherapy. Cancer. 2003;98(7):1521-30. https://doi.org/10.1002/cncr.11660.

16. Imamoto H, Oba K, Sakamoto J, Iishi H, Narahara H, Yumiba T, et al. Assessing clinical benefit response in the treatment of gastric malignant ascites with non-measurable lesions: a multicenter phase II trial of paclitaxel for malignant ascites secondary to advanced/recurrent gastric cancer. Gastric Cancer. 2011;14(1):8190. https://doi.org/10.1007/s10120-011-0016-6.

17. Yonemura Y, Bandou E, Sawa T, Yoshimitsu Y, Endou Y, Sasaki $\mathrm{T}$, et al. Neoadjuvant treatment of gastric cancer with peritoneal dissemination. Eur J Surg Oncol. 2006;32(6):661-5. https://doi. org/10.1016/j.ejso.2006.03.007.

18. Yonemura Y, Endou Y, Shinbo M, Sasaki T, Hirano M, Mizumoto A, et al. Safety and efficacy of bidirectional chemotherapy for treatment of patients with peritoneal dissemination from gastric cancer: selection for cytoreductive surgery. J Surg Oncol. 2009;100(4):311-6. https://doi.org/10.1002/jso.21324.

19. Badgwell B, Blum M, Das P, Estrella J, Wang X, Ho L, et al. Phase II trial of laparoscopic hyperthermic intraperitoneal chemoperfusion for peritoneal carcinomatosis or positive peritoneal cytology in patients with gastric adenocarcinoma. Ann Surg Oncol. 2017;24(11):3338-44. https://doi.org/10.1245/s1043 4-017-6047-4.

20. Takeshita K, Liu Y, Ishibashi H, Yonemura Y. Laparoscopic hyperthermic intraperitoneal chemotherapy for peritoneal carcinomatosisfrom gastric cancer: its beneficial effects on reduction and exact evaluation of the peritoneal cancer index. Am Surg. 2017;83(11):1315-20.

21. Nadiradze G, Giger-Pabst U, Zieren J, Strumberg D, Solass W, Reymond MA. Pressurized intraperitoneal aerosol chemotherapy (PIPAC) with low-dose cisplatin and doxorubicin in gastric peritoneal metastasis. J Gastrointest Surg. 2016;20(2):367-73. https://doi.org/10.1007/s11605-015-2995-9.

22. Canbay E, Mizumoto A, Ichinose M, Ishibashi H, Sako S, Hirano M, et al. Outcome data of patients with peritoneal carcinomatosis from gastric origin treated by a strategy of bidirectional chemotherapy prior to cytoreductive surgery and hyperthermic intraperitoneal chemotherapy in a single specialized center in Japan. Ann Surg Oncol. 2014;21(4):1147-52. https:// doi.org/10.1245/s10434-013-3443-2.

23. Yoshida M, Sugino T, Kusafuka K, Nakajima T, Makuuchi $\mathrm{R}$, Tokunaga $\mathrm{M}$, et al. Peritoneal dissemination in early gastric cancer: importance of the lymphatic route. Virchows Arch. 2016;469(2):155-61. https://doi.org/10.1007/s0042 8-016-1960-7.

24. Smyth EC, Fassan M, Cunningham D, Allum WH, Okines AF, Lampis A, et al. Effect of pathologic tumor response and nodal status on survival in the medical research council adjuvant gastric infusional chemotherapy trial. J Clin Oncol. 2016;34(23):2721-7. https://doi.org/10.1200/jco.2015.65.7692 (http://www.jco.org. Author contributions are found at the end of this article).

25. Stillwell AP, Ho YH, Veitch C. Systematic review of prognostic factors related to overall survival in patients with stage IV colorectal cancer and unresectable metastases. World J Surg. 2011;35(3):684-92. https://doi.org/10.1007/s00268-010-0891-8.

26. An X, Sarmiento C, Tan T, Zhu H. Regulation of multidrug resistance by microRNAs in anti-cancer therapy. Acta Pharm Sin B. 2017;7(1):38-51. https://doi.org/10.1016/j.apsb.2016.09.002.

27. Gong J, Jaiswal R, Mathys JM, Combes V, Grau GE, Bebawy M. Microparticles and their emerging role in cancer multidrug resistance. Cancer Treat Rev. 2012;38(3):226-34. https://doi. org/10.1016/j.ctrv.2011.06.005.

28. Yang W, Ma J, Zhou W, Cao B, Zhou X, Yang Z, et al. Molecular mechanisms and theranostic potential of miRNAs in drug resistance of gastric cancer. Expert Opin Ther Targets. 2017;21(11):1063-75. https://doi.org/10.1080/14728 222.2017.1389900.

29. Ji R, Zhang B, Zhang X, Xue J, Yuan X, Yan Y, et al. Exosomes derived from human mesenchymal stem cells confer drug resistance in gastric cancer. Cell Cycle. 2015;14(15):2473-83. https:// doi.org/10.1080/15384101.2015.1005530.

30. Kurokawa Y, Hamakawa T, Miyazaki Y, Takahashi T, Yamasaki M, Miyata $\mathrm{H}$, et al. Preoperative systemic and intraperitoneal chemotherapy consisting of S-1, cisplatin and docetaxel in patients with marginally resectable gastric cancer. Anticancer Res. 2015;35(4):2223-8.

31. Al-Batran SE, Homann N, Pauligk C, Illerhaus G, Martens UM, Stoehlmacher J, et al. Effect of neoadjuvant chemotherapy followed by surgical resection on survival in patients with limited metastatic gastric or gastroesophageal junction cancer: the AIOFLOT3 Trial. JAMA Oncol. 2017;3(9):1237-44. https://doi. org/10.1001/jamaoncol.2017.0515.

32. Ott K, Sendler A, Becker K, Dittler HJ, Helmberger H, Busch R, et al. Neoadjuvant chemotherapy with cisplatin, 5-FU, and leucovorin (PLF) in locally advanced gastric cancer: a prospective phase II study. Gastric Cancer. 2003;6(3):159-67. https://doi. org/10.1007/s10120-003-0245-4.

33. Achilli P, De Martini P, Ceresoli M, Mari GM, Costanzi A, Maggioni D, et al. Tumor response evaluation after neoadjuvant chemotherapy in locally advanced gastric adenocarcinoma: a prospective, multi-center cohort study. J Gastrointest Oncol. 2017;8(6):1018-25. https://doi.org/10.21037/jgo.2017.08.13.

34. Los G, Mutsaers PH, van der Vijgh WJ, Baldew GS, de Graaf PW, McVie JG. Direct diffusion of cis-diamminedichloroplatinum(II) in intraperitoneal rat tumors after intraperitoneal chemotherapy: a comparison with systemic chemotherapy. Cancer Res. 1989;49(12):3380-4. 
35. Yang XJ, Huang CQ, Suo T, Mei LJ, Yang GL, Cheng FL, et al. Cytoreductive surgery and hyperthermic intraperitoneal chemotherapy improves survival of patients with peritoneal carcinomatosis from gastric cancer: final results of a phase III randomized clinical trial. Ann Surg Oncol. 2011;18(6):1575-81. https://doi. org/10.1245/s10434-011-1631-5.

36. Rau B. Prospective multicentric phase III study. Z Gastroenterol. 2014;52(3):262.

37. Sunakawa Y, Stremitzer S, Cao S, Zhang W, Yang D, Wakatsuki $\mathrm{T}$, et al. Association of variants in genes encoding for macrophage-related functions with clinical outcome in patients with locoregional gastric cancer. Ann Oncol. 2015;26(2):332-9. https ://doi.org/10.1093/annonc/mdu542.
38. Fukuhara S, Yabe M, Montgomery MM, Itagaki S, Brower ST, Karpeh MS Jr. Race/ethnicity is predictive of lymph node status in patients with early gastric cancer. J Gastrointest Surg. 2014;18(10):1744-51. https://doi.org/10.1007/s1160 5-014-2590-5

Publisher's Note Springer Nature remains neutral with regard to jurisdictional claims in published maps and institutional affiliations. 